\title{
Efecto del sistema y densidad de la almaciguera en el cultivo de cebolla (Allium cepa)
}

\author{
César Gómez y ${ }^{1}$ Christel Oberpaur ${ }^{1}$ \\ Escuela de Agronomía, Universidad Santo Tomás, Ejército 146, Santiago, Chile.
}

\begin{abstract}
C. Gómez, and C. Oberpaur. 2007. Effect of density and seedbed system in onion (Allium cepa) production. Cien. Inv. Agr. 34(3):205-214. Production of onion (Allium cepa) seedlings for transplants is done directly in flat soil conditions or in trays using reduced cell volumes and high plant density conditions, without consideration of the possible effect that reduced cell volume can have on crop growth. Therefore, the objective of this study was to evaluate the production of onion seedlings in two seedbed systems and their subsequent behavior in the field. Seedling production and transplant establishment were determined with a long-day type of onion (cv. Grano de Oro) in the Metropolitan Region of Chile ( $33^{\circ}-34^{\circ}$ south latitude) from 2004 to 2005 . The production of covered root transplants was performed in trays with 286 (18 $\left.\mathrm{cm}^{3}\right), 432\left(10 \mathrm{~cm}^{3}\right)$ and $814\left(5 \mathrm{~cm}^{3}\right)$ cells each, equivalent to plant densities of 1144 (low), 1728 (medium) and 3256 (high) plants $\mathrm{m}^{-2}$, respectively. The production of bare root transplants was done in boxes with equal plant densities as in the tray system. Next, plants obtained from each seedbed system and plant density were field transplanted. Based on the results obtained, neither plant density nor transplant production system affected the pseudo stem diameter 25 days after emergency (DAE). At 77 DAE, the pseudo stem diameters were 3.8 and $5.2 \mathrm{~mm}$, the root dry weights (DW) were 4.8 and $6.2 \mathrm{~g} \cdot$ plant $^{-1}$ produced in high and low plant density, respectively. This suggests that the tray system had a minor effect on plant development. At 23 days after transplanting (DAT) differences in the pseudo stem diameter between the three densities were obtained, although differences between the bare and covered root systems were not statistically significant. Plants obtained from high-density seedbeds showed a smaller pseudo stem diameter and lower canopy DW than plants produced in low-density seedbeds until 84 DAT. At harvest, no differences were observed in formation or among the quality categories of the onion. Therefore, it is feasible to produce onion seedlings to be transplanted with bare roots or covered roots, produced in high-density boxes or in $5 \mathrm{~cm}^{3}$ cells, without affecting the final quality of harvested onion bulbs.
\end{abstract}

Key words: Onion production, seedling density, seedling production.

\section{Introducción}

Al analizar las plantaciones de cebollas chilenas, aproximadamente el $20 \%$ de los almácigos carece del mínimo estándar de calidad, pues su calibre está bajo del diámetro requerido a nivel del cuello. Esto representa una gran desventaja para alcanzar un rápido y vigoroso reestablecimiento después del trasplante (Aljaro, 2001b). Aunque se dispone

Recibido 23 enero 2007. Aceptado 31 agosto 2007.

'Dirigir correspondencia a C. Oberpaur: coberpaur@santotomas.cl de las condiciones naturales para alcanzar altos rendimientos y calidad, existen pocos antecedentes sobre sistemas de almácigos y contenedores más efectivos que podrían mejorar la productividad del cultivo (Aljaro, 2001a).

En la producción de plantines de cebollas se utilizan almacigueras tradicionales, realizadas directamente en el suelo, en camas o en mesas, o bien en diversos tipos de contenedores. En el primer caso el traslado de los plantines al lugar definitivo se hace siempre a raíz desnuda, y en el segundo, dependiendo del contenedor, se transplanta a raíz desnuda (cajoneras) o a raíz 
cubierta (bandejas con alvéolos individuales). Al usar este último sistema, los plantines se trasladan con su sistema radical intacto.

La elección del tamaño de alvéolo o la densidad de siembra a menudo se decide de acuerdo con principios económicos. Sin embargo, las tasas de crecimiento tienden a ser proporcionales al volumen del alvéolo; mientras mayor es el espacio disponible para cada plantín, mayor tamaño alcanza. Cuando se prolonga en demasía el tiempo de permanencia del plantín en un cierto tamaño de alvéolo, se aumenta progresivamente la restricción del crecimiento tanto radical como aéreo (Wien, 1997).

Los alvéolos más grandes se recomiendan para plantines con ciclos vegetativos relativamente más largos ( $>5$ semanas), mientras que los alvéolos más pequeños pueden ser la mejor opción para las hortalizas de ciclo corto $(<4$ semanas). Las cebollas, permanecen en el invernadero por un período más largo (10 a 12 semanas) que la mayoría de los otros cultivos. Sin embargo, se prefieren contenedores con alvéolos pequeños, debido a la gran cantidad de plantas por hectárea requeridas para la producción comercial (Vavrina, 2002)

Numerosas investigaciones realizadas sobre distintas especies hortícolas, han estudiado el volumen óptimo para obtener un plantín de calidad. En general, la mayoría de los estudios concluyen que los plantines más vigorosos, de mayor desarrollo radical y precocidad, se obtienen en alvéolos de mayor volumen (Marsh y Paul, 1988; Wien 1997). En cebollas, se obtienen plantas de calidad en alvéolos de 4 a $7 \mathrm{~cm}^{3}$, en un período de 8 a 10 semanas (Reynauld et al., 1999). El objetivo de este estudio fue evaluar la producción de plantines de cebolla en dos sistemas de almaciguera y su posterior comportamiento en campo hasta la cosecha de los bulbos.

\section{Materiales y métodos}

Se efectuaron dos ensayos, el primero de almaciguera, realizado en un invernadero comercial calefaccionado (temperatura mínima media $10^{\circ} \mathrm{C}$ y máxima media $27^{\circ} \mathrm{C}$ ) ubicado en Talagante, y el segundo de trasplante y cultivo efectuado al aire libre (temperatura mínima media $8,5^{\circ} \mathrm{C}$, y máxima media $25,4^{\circ} \mathrm{C}$ ) en Santiago. Ambas localidades de la Región Metropolitana ubicadas entre los $33^{\circ}$ y $34^{\circ}$ lat. sur.

En los dos ensayos se utilizaron semillas de cebolla cv. Grano de Oro (Seminis). Este cultivar corresponde a cebolla de día largo para guarda, que presenta un período vegetativo entre 120 y 130 días, medido desde trasplante a cosecha.

En el ensayo de almácigo en invernadero se ocuparon 12 bandejas de poliestireno, de $5 \mathrm{x}$ 39 x $64 \mathrm{~cm}$, con 286, 432 y 814 alvéolos por contenedor, cada una con alvéolos de 18, 10 y $5 \mathrm{~cm}^{3}$, respectivamente. Se emplearon cuatro bandejas de cada tipo. Paralelamente se usaron 12 cajoneras de poliestireno, de $10 \times 39$ × 64 $\mathrm{cm}$, con una altura de $5 \mathrm{~cm}$ de sustrato, las que se emplearon para almácigos para transplantar a raíz desnuda. Como sustrato se ocupó una mezcla comercial (Sungro Sunshine 3), basada en turba, de origen canadiense, yeso, cal dolomita, vermiculita y un agente humectante.

\section{Ensayo 1. Crecimiento de plantines}

La siembra se realizó manualmente el 15 de julio de 2004, colocando una semilla por alvéolo en el caso de los contenedores. En las cajoneras las semillas se distribuyeron de modo de lograr una densidad equivalente a los contenedores, es decir 286, 432 y 814 semillas por cajonera. El riego se realizó por micro aspersión, aplicándose por esta vía, los fertilizantes y los fungicidas. Antes del trasplante, los plantines se llevaron a condiciones de aire libre por un período de 8 días, con el propósito de adaptarlas a las temperaturas externas.

\section{Ensayo 2. Trasplante, desarrollo y crecimiento en campo}

La preparación del suelo para el trasplante se efectuó en forma mecanizada y el afinamiento se realizó con rastrillo. El trasplante se realizó a las 12 semanas (10 de octubre de 2004), cuando las raíces del almácigo en contenedores llenaron el alvéolo, sin presentar oxidaciones y, en lo posible, cuando coincidiera con un diámetro de 
Cuadro 1. Tratamientos aplicados en almaciguera y transplante de cebolla cv. Grano de Oro. Región Metropolitana, 2004-2005.

Table 1. Treatments applied at transplant production and subsequent transplanting of onion cv. Grano de Oro. Metropolitan Region, Chile, 2004-2005.

\begin{tabular}{|c|c|c|c|}
\hline Tipo de almaciguera & $\begin{array}{l}\text { Alvéolos por contenedor } \\
\text { no. }\end{array}$ & $\begin{array}{l}\text { Volumen de celda } \\
\mathrm{cm}^{3}\end{array}$ & $\begin{array}{l}\text { Densidad } \\
\text { planta }\left(\mathrm{m}^{2}\right)^{-1}\end{array}$ \\
\hline \multicolumn{4}{|c|}{ Trasplante a raíz cubierta $(T R C)$ : } \\
\hline \multirow[t]{3}{*}{ Contenedor ${ }^{1}$} & 286 & 18 & 1144 \\
\hline & 432 & 10 & 1728 \\
\hline & 814 & 5 & 3256 \\
\hline \multicolumn{4}{|c|}{ Trasplante a raíz desnuda (TRD): } \\
\hline \multirow{3}{*}{ Cajonera $^{2}$} & & & 1144 \\
\hline & & & 1728 \\
\hline & & & 3256 \\
\hline
\end{tabular}

5 a $6 \mathrm{~mm}$ a nivel de cuello en ambos tipos de almaciguera.

Se seleccionaron plantas uniformes en tamaño del Ensayo 1, las que se establecieron en parcelas de $6,6 \mathrm{~m}^{2}$, sobre mesas de $70 \mathrm{~cm}$ de ancho, con cuatro hileras de 55 plantas y 220 plantas por repetición. Se plantaron a $10 \mathrm{~cm}$ sobre la hilera y $15 \mathrm{~cm}$ entre hileras, con una densidad de plantación equivalente a 333.333 plantas $\cdot \mathrm{ha}^{-1}$. El riego se realizó por cintas y el control de malezas se efectuó manualmente. En el Cuadro 1 se indican los tratamientos utilizados en la etapa de almaciguera (Ensayo 1) y posterior trasplante (Ensayo 2).

En ambos ensayos, el efecto del sistema de producción de plantines se evaluó por los siguientes parámetros: 1 . Peso seco de raíces $\left(72^{\circ} \mathrm{C}\right.$ por $\left.24 \mathrm{~h}\right)$, diámetro del cuello, peso seco de la canopia $\left(90^{\circ} \mathrm{C}\right.$ por $\left.24 \mathrm{~h}\right)$, y altura de la planta, en muestreos completamente al azar de ocho plantas por repetición. En los almácigos, las muestras se tomaron quincenalmente, pero el peso seco de la canopia y la altura de planta se evaluó sólo 10 y 25 después de emergencia (dde). Posteriormente se efectuó una chapoda, práctica habitual entre los viveristas, que favorece la aireación entre los plantines (Aljaro, 2001a). En el segundo ensayo las mediciones se realizaron al momento del trasplante $y$, posteriormente, cada 30 días hasta cosecha.
La cosecha de bulbos se realizó el 3 de febrero de 2005, momento en que se determinó el diámetro ecuatorial del bulbo fresco y presencia de malformaciones en 50 plantas al azar. Las plantas sin malformaciones se clasificaron en las siguientes por categoría según el diámetro del bulbo: $<75 \mathrm{~mm}, 76-90 \mathrm{~mm}$ y $>91 \mathrm{~mm}$.

\section{Diseño y análisis estadísticos}

El Ensayo 1 se diseñó en forma completamente al azar con estructura factorial, 2 x 3 (dos tipos de contenedores, alvéolo y cajonera, cada una con tres densidades de plantas), y cuatro repeticiones de un contenedor o cajonera cada una.

El Ensayo 2 se diseñó en bloques completos al azar con estructura factorial, 2 × 3 (tipo de almaciguera, alvéolo o cajonera, y tres densidades de planta), y cuatro repeticiones de 220 plantas como unidad experimental.

Los resultados obtenidos en ambos ensayos se sometieron a un análisis de varianza y los promedios se separaron según la prueba de Tukey $(\mathrm{p}<0,05)$. Los valores porcentuales se transformaron según Bliss antes de los análisis estadísticos. Con este propósito se empleó el software Minitab Stadistical, Release 13.31 (EUA). 


\section{Resultados}

\section{Ensayo 1. Crecimiento de plantines}

A los 10 días después de emergencia (dde), cuando las plantas alcanzaron el estado de hoja bandera, la densidad de plantas tuvo un efecto significativo $(\mathrm{p}<0,0001)$ sobre el diámetro del cuello (Cuadro 5). No obstante, las distintas densidades ensayadas tuvieron un efecto errático, siendo imposible establecer una asociación directa entre ambos factores. La interacción entre las diferentes densidades y el tipo de almaciguera fue estadísticamente significativa $(\mathrm{p}<0,0001)$ (Cuadro 2).

A los 25 dde, el sistema de producción de almácigos tuvo un efecto significativo ( $\mathrm{p}<$ $0,0001)$ y a su vez hubo diferencias significativas $(\mathrm{p}<0,05)$ entre los sistemas de producción de almácigos, siendo los almácigos producidos en contenedores superiores en diámetro de plantas que los producidos en cajoneras. Sin embargo, no hubo diferencias significativas entre densidades de siembra, y la interacción entre ambos factores tampoco fue estadísticamente significativa (Cuadros 2, 5).

La mayor densidad de siembra afectó negativamente, tanto en las bandejas como en las cajoneras, luego de los 40 dde. A los 55 dde, se observó diferencias significativas $(\mathrm{p}<0,05)$ entre las tres densidades de siembra ensayadas, no así entre los sistemas de producción de almácigos (Cuadros 2, 5). En ambos sistemas de producción, la densidad mas baja favoreció un mayor diámetro de planta. La interacción entre los factores no fue estadísticamente significativa.

A los 77 dde, la densidad de planta y el tipo de almaciguera tuvieron un efecto significativo sobre el diámetro del cuello, pero sólo la densidad de planta tuvo un efecto significativo sobre el peso radical (Cuadros 2, 5). Se obtuvieron mayores diámetros de cuello de los

Cuadro 2. Efecto de la densidad y tipo de almaciguera sobre el diámetro del cuello, y el peso seco radical en cebolla cv. Grano de Oro obtenido después de 10, 25, 40, 55 y 77 días de la emergencia. Talagante, 2004.

Table 2. Effect of density and transplant production system on pseudo stem diameter and root dry weight of onion cv. Grano de Oro obtained after 10, 25, 40, 55 and 77 days of emergency. Talagante, 2004.

\begin{tabular}{|c|c|c|c|c|c|}
\hline \multirow{2}{*}{$\begin{array}{l}\text { Días } \\
\text { después del } \\
\text { transplante }\end{array}$} & \multirow{2}{*}{$\begin{array}{l}\text { Densidad } \\
\text { planta }\left(\mathrm{m}^{2}\right)^{-1}\end{array}$} & \multicolumn{2}{|c|}{ Diámetro del cuello, mm } & \multicolumn{2}{|c|}{ Peso seco radical, mg $\cdot$ planta $^{-1}$} \\
\hline & & $\begin{array}{l}\text { Contenedor } \\
\text { raíz cubierta }\end{array}$ & $\begin{array}{l}\text { Cajonera } \\
\text { raíz desnuda }\end{array}$ & $\begin{array}{l}\text { Contenedor } \\
\text { raíz cubierta }\end{array}$ & $\begin{array}{l}\text { Cajonera } \\
\text { raíz desnuda }\end{array}$ \\
\hline \multirow[t]{3}{*}{10} & 1144 & $0,73 \mathrm{a} \mathrm{A}^{1}$ & 0,76 a $A^{1}$ & nd & nd \\
\hline & 1728 & 0,69 b A & 0,64 b B & nd & nd \\
\hline & 3256 & 0,71 a $\mathrm{B}$ & 0,77 a $\mathrm{A}$ & nd & nd \\
\hline \multirow[t]{3}{*}{25} & 1144 & 1,77 a $\mathrm{A}$ & 1,47 a B & 9 a $\mathrm{A}$ & 6 a $\mathrm{A}$ \\
\hline & 1728 & 1,69 a $\mathrm{A}$ & 1,45 a B & 6 a $\mathrm{A}$ & 5 a $\mathrm{A}$ \\
\hline & 3256 & 1,65 a A & 1,46 a B & 7 a $\mathrm{A}$ & 4 a $\mathrm{A}$ \\
\hline \multirow[t]{3}{*}{40} & 1144 & 2,97 a A & 2,22 a B & 26 a $\mathrm{A}$ & 18 a $\mathrm{A}$ \\
\hline & 1728 & 2,62 a A & 2,31 a B & $19 \mathrm{abA}$ & $16 \mathrm{abA}$ \\
\hline & 3256 & $2,20 \mathrm{~b} \mathrm{~A}$ & 1,94 b B & $14 \mathrm{~b} \mathrm{~A}$ & $15 \mathrm{~b} \mathrm{~A}$ \\
\hline \multirow[t]{3}{*}{55} & 1144 & 3,49 a $\mathrm{A}$ & 3,21 a $\mathrm{A}$ & 58 a $\mathrm{A}$ & 43 a $\mathrm{A}$ \\
\hline & 1728 & $2,94 \mathrm{~b} \mathrm{~A}$ & $2,93 \mathrm{~b} \mathrm{~A}$ & $33 \mathrm{~b} \mathrm{~A}$ & $40 \mathrm{~b} \mathrm{~A}$ \\
\hline & 3256 & $2,39 \mathrm{c} \mathrm{A}$ & $2,72 \mathrm{c} \mathrm{A}$ & $22 \mathrm{c} \mathrm{A}$ & $35 \mathrm{c} \mathrm{A}$ \\
\hline \multirow[t]{3}{*}{77} & 1144 & 5,24 a B & 6,16 a $A$ & 136 a A & 128 a A \\
\hline & 1728 & 4,63 a B & 5,74 a A & 99 a $\mathrm{A}$ & $111 \mathrm{abA}$ \\
\hline & 3256 & 3,78 b B & $4,81 \mathrm{~b} \mathrm{~A}$ & $61 \mathrm{~b} \mathrm{~A}$ & $88 \mathrm{~b} \mathrm{~A}$ \\
\hline
\end{tabular}

${ }^{1}$ Promedios unidos por letras minúsculas y mayúsculas iguales indican diferencias estadísticamente no significativas entre densidades de plantación (columnas) y entre tipo de almacigueras (filas), respectivamente, según la prueba de rangos múltiples de Tukey (p <0,05). Nd, no determinado.

${ }^{\prime}$ Mean values followed by similar small and capital letters indicate no statistical differences in relation to plant densities (columns) and among container types (rows) according to Tukey test $(p=0.05)$. 
Cuadro 3. Efecto de la densidad y tipo de almaciguera sobre el diámetro del cuello, peso seco radical y peso seco aéreos obtenido 23 y 53 días después del transplante en cebolla cv. Grano de Oro. Santiago, 2004-2005.

Table 3. Effect of density and transplant production system on pseudo stem diameter, root dry weight and canopy dry weight obtained 23 and 55 days after transplanting date on onion cv. Grano de Oro. Santiago, 2004-2005.

\begin{tabular}{|c|c|c|c|c|c|c|}
\hline \multirow[b]{2}{*}{$\begin{array}{l}\text { Densidad } \\
\text { planta }\left(\mathrm{m}^{2}\right)^{-1}\end{array}$} & \multicolumn{2}{|c|}{$\begin{array}{c}\text { Diámetro del cuello } \\
\mathrm{mm}\end{array}$} & \multicolumn{2}{|c|}{$\begin{array}{c}\text { Peso seco radical } \\
\mathrm{mg} \cdot \text { planta }^{-1}\end{array}$} & \multicolumn{2}{|c|}{$\begin{array}{c}\text { Peso seco aéreo } \\
\mathrm{mg} \cdot \text { planta }^{-1}\end{array}$} \\
\hline & $\begin{array}{l}\text { Contenedor } \\
\text { raíz cubierta }\end{array}$ & $\begin{array}{l}\text { Cajonera } \\
\text { raíz desnuda }\end{array}$ & $\begin{array}{l}\text { Contenedor } \\
\text { raíz cubierta }\end{array}$ & $\begin{array}{l}\text { Cajonera } \\
\text { raíz desnuda }\end{array}$ & $\begin{array}{l}\text { Contenedor } \\
\text { raíz cubierta }\end{array}$ & $\begin{array}{c}\text { Cajonera } \\
\text { raíz desnuda }\end{array}$ \\
\hline \multicolumn{7}{|c|}{23 días después del transplante: } \\
\hline 1144 & 7,58 a A & 7,76 a $\mathrm{A}$ & 512 a $\mathrm{A}$ & 453 a A & 608 a $\mathrm{A}$ & 660 a $A$ \\
\hline 1728 & $6,69 \mathrm{~b} \mathrm{~A}$ & $6,69 \mathrm{~b} \mathrm{~A}$ & 380 a $\mathrm{A}$ & 448 a A & $418 \mathrm{~b} \mathrm{~A}$ & $480 \mathrm{~b} \mathrm{~A}$ \\
\hline 3256 & 5,33 с A & $5,66 \mathrm{c} \mathrm{A}$ & 288 a A & 305 a A & $278 \mathrm{c} \mathrm{A}$ & 348 c A \\
\hline \multicolumn{7}{|c|}{53 días después del transplante: } \\
\hline 1144 & 15,37 a A & 15,06 a A & $1.152 \mathrm{a} \mathrm{A}$ & 1.118 a A & $3.235 \mathrm{a} \mathrm{A}$ & $3.010 \mathrm{a} \mathrm{A}$ \\
\hline 1728 & 14,06 a A & 13,77 a A & $1.090 \mathrm{a} \mathrm{A}$ & 791 a A & $2.495 \mathrm{ab} \mathrm{A}$ & $2.645 \mathrm{~b} \mathrm{~A}$ \\
\hline 3256 & 13,68 a $\mathrm{A}$ & 12,94 a A & 829 a A & 762 a A & 2.148 b A & $2.393 \mathrm{~b} \mathrm{~A}$ \\
\hline
\end{tabular}

${ }^{1}$ Promedios unidos por letras minúsculas y mayúsculas iguales indican diferencias estadísticamente no significativas entre densidades de plantación (columnas) y entre tipo de almacigueras (filas), respectivamente, según la prueba de rangos múltiples de Tukey ( $\mathrm{p}=0,05$ ).

${ }^{I}$ Mean values followed by similar small and capital letters indicate no statistical differences in relation to plant densities (columns) and among container types (rows) according to Tukey test $(p=0.05)$.

Cuadro 4. Efecto de la densidad y tipo de almaciguera sobre el diámetro del bulbo fresco, presencia de bulbos malformados y proporción de bulbos exportables con calibres superiores a $76 \mathrm{~mm}$, obtenidos a la cosecha de cebollas cv. Grano de Oro, 114 días después del transplante. Santiago, 2005

Table 4. Effect of density and transplant production system on fresh bulb diameter, presence of deformed bulbs (\%) and proportion of exportable bulbs with size over 76 mm obtained at harvest time of onions $\mathrm{cv}$. Grano de Oro, 114 days after transplanting. Santiago, 2005.

\begin{tabular}{ccc}
\hline \multirow{2}{*}{$\begin{array}{c}\text { Densidad en } \\
\text { almaciguera } \\
\text { plantas } \mathrm{m}^{-2}\end{array}$} & \multicolumn{2}{c}{ Tipo de almaciguera } \\
\cline { 2 - 3 } & $\begin{array}{c}\text { Contenedor } \\
\text { raíz cubierta }\end{array}$ & $\begin{array}{c}\text { Cajonera } \\
\text { raíz desnuda }\end{array}$ \\
\hline $\begin{array}{c}\text { Diámetro del bulbo, cm } \\
1144\end{array}$ & 8,97 a A & 9,00 a A \\
1728 & 8,83 a A & 8,09 a A \\
3256 & 8,38 a A & 8,27 a A \\
\hline Bulbos malformados, \% & & \\
1144 & 19,5 a A & 15,0 a A \\
1728 & 10,5 a A & 10,0 a A \\
3256 & 9,5 a A & 19,5 a A \\
\hline Bulbos exportables, \% & & \\
1144 & 91,5 a A & 89,0 a A \\
1728 & 92,5 a A & 81,5 a A \\
3256 & 77,5 a A & 74,0 a A \\
\hline
\end{tabular}

${ }^{1}$ Promedios unidos por letras minúsculas y mayúsculas iguales indican diferencias estadísticamente no significativas entre densidades en almaciguera (columnas) y entre tipo de almacigueras (filas), respectivamente, según la prueba de rangos múltiples de Tukey $(\mathrm{p}=0,05)$.

${ }^{\prime}$ Mean values followed by similar small and capital letters indicate no statistical differences in relation to nursery plant densities (columns) and among container types (rows) according to Tukey test $(p=0.05)$. plantines sembrados en cajonera. En ambos sistemas de producción la densidad de planta más alta tuvo un mayor efecto restrictivo. Tampoco la interacción entre el tipo producción y la densidad de planta de la almaciguera fue significativa.

En relación al peso seco radical, no se detectó diferencias significativas entre las densidades de planta y los tipos de almacigueras estudiados a los 10 dde. El tipo de almaciguera y la densidad de planta afectaron el peso seco de la raíz después de los 25 dde, momento en que el volumen de la celda no estuvo completamente ocupado por el sistema radical. Sin embargo, la mayor densidad restringió el crecimiento radical, en ambos sistemas de almaciguera a los 40 dde. A los 55 dde, el peso seco radical presentó diferencias significativas $(\mathrm{p}<0,05)$ entre las densidades de siembra, pero los tipos de almaciguera no tuvieron un efecto significativo. El efecto de la densidad de plantación y el tipo de almaciguera sobre el peso seco radical, a los 25, 40, 55 y 77 dde se indica en el Cuadro 2.

La evaluación del peso seco radical determinado a los 77 dde mostró la misma tendencia que el diámetro del cuello. Con la menor densidad de planta, principalmente en contenedores, siempre se obtuvo un mayor peso seco radical. Las densidades de planta medias y altas 
mostraron un aumento de peso prácticamente similar entre si hasta los 40 dde. Recién a los 55 dde las diferencias en peso seco radical fueron significativas $(p<0,05)$ entre las tres densidades de plantación estudiadas. A los 77 dde, la densidad más alta afectó notoriamente el peso radical seco, sobre todo en plantines provenientes de alvéolos individuales (Cuadro 2). No hubo interacción significativa entre los factores estudiados en relación con el peso radical en ninguna de las fechas de evaluación (Cuadro 5).

Con relación al peso seco aéreo de las plantas, ni el sistema de producción ni la densidad de almaciguera tuvieron un efecto significativo, alcanzando un peso de $3 \mathrm{mg} \cdot$ planta $^{-1}$ a los 10 dde. A los 25 dde, el tipo de almaciguera tuvo un efecto significativo sobre el peso aéreo seco ( $\mathrm{p}<0,0001$ ) (Cuadros 2, 5). Las plántulas provenientes de contenedores pesaron más que las obtenidas en cajoneras con 10 a 11 y $8 \mathrm{mg} \cdot$ planta $^{-1}$, respectivamente. No hubo diferencias estadísticamente significativas entre densidades de planta y la interacción entre estos factores tampoco fue significativa (Cuadro 5).

En las plantas provenientes de contenedores la altura varió entre 7,1 y $7,3 \mathrm{~cm}$, y las plantas producidas en cajoneras variaron entre 6,4 y $6,8 \mathrm{~cm}$ a los 25 dde. Sin embargo, en este parámetro no hubo diferencias estadísticamente significativas en ninguno de los tratamientos estudiados (datos no presentados).

\section{Ensayo 2. Trasplante, desarrollo y crecimiento en terreno}

Se obtuvo un $100 \%$ de plantas establecidas en los tratamientos a los 15 días después del trasplante (ddt). Esto indica que la densidad de siembra y el tipo de almaciguera no influyeron en la supervivencia de las plantas en terreno.

Cuadro 5. Análisis de varianza del ensayo en almaciguera y del ensayo transplantado, hasta cosecha, de cebolla cv. Grano de Oro. 2004-2005.

Table 5. Analisis of variance of a plant nursery trial and a transplanted trial of onion cv. Grano de Oro. 2004-2005.

\begin{tabular}{|c|c|c|c|c|c|c|c|c|c|c|}
\hline \multirow[t]{3}{*}{ Causa de variación } & \multicolumn{5}{|c|}{$\begin{array}{l}\text { Diámetro del cuello } \\
\text { días }\end{array}$} & \multicolumn{3}{|c|}{$\begin{array}{l}\text { Peso radical }{ }^{1} \\
\text { días }\end{array}$} & \multicolumn{2}{|c|}{$\begin{array}{l}\text { Peso aéreo }^{1} \\
\text { días }\end{array}$} \\
\hline & 10 & 25 & 40 & 55 & 77 & 25 & 40 & 55 & 77 & 25 \\
\hline & $\mathrm{p}$ & $\mathrm{p}$ & $\mathrm{p}$ & $\mathrm{p}$ & $\mathrm{p}$ & $\mathrm{p}$ & $\mathrm{p}$ & $\mathrm{p}$ & $\mathrm{p}$ & $\mathrm{p}$ \\
\hline \multicolumn{11}{|l|}{ Almaciguera: } \\
\hline Densidad (D) $\quad<$ & $<0,0001$ & 0,633 & 0,002 & $<0,0001$ & $<0,0001$ & 0,369 & 0,008 & 0,001 & 0,001 & 0,941 \\
\hline Tipo almaciguera (TA) & 0,152 & $<0.0001$ & 0,001 & 0,871 & $<0,0001$ & 0,242 & 0,176 & 0,109 & 0,340 & $<0,0001$ \\
\hline Interacción D x TA $<$ & $<0,0001$ & 0,710 & 0,143 & 0,108 & 0,946 & 0,449 & 0,166 & 0,108 & 0,430 & 0,615 \\
\hline \multirow[t]{3}{*}{ Post trasplante: } & \multicolumn{3}{|c|}{ días } & & & \multicolumn{3}{|c|}{ días } & \multicolumn{2}{|c|}{ días } \\
\hline & 23 & & 53 & & & 23 & & 53 & 23 & 53 \\
\hline & $\mathrm{p}$ & \multicolumn{2}{|c|}{$\mathrm{p}$} & & & $\mathrm{p}$ & & $\mathrm{p}$ & $\mathrm{p}$ & $\mathrm{p}$ \\
\hline Densidad (D) & \multicolumn{2}{|l|}{$<0,0001$} & \multicolumn{3}{|c|}{0,387} & 0,072 & & 0,225 & $<0,0001$ & 0,008 \\
\hline Tipo almaciguera (TA) & 0,501 & & 331 & & & 0,966 & & 0,398 & 0,183 & 0,772 \\
\hline \multirow[t]{3}{*}{ Interacción D x TA } & 0,871 & & 387 & & & 0,604 & & 0,747 & 0,987 & 0,583 \\
\hline & \multicolumn{3}{|c|}{$\begin{array}{c}\text { Diámetro del bulbo } \\
114 \text { días }\end{array}$} & $\begin{array}{l}\text { os malform } \\
114 \text { días }\end{array}$ & ados & \multicolumn{3}{|c|}{$\begin{array}{c}\text { Bulbos exportables } \\
114 \text { días }\end{array}$} & & \\
\hline & \multicolumn{3}{|c|}{$\mathrm{p}$} & $\mathrm{p}$ & & \multicolumn{3}{|c|}{$\mathrm{p}$} & & \\
\hline Densidad (D) & \multicolumn{3}{|c|}{0,063} & 0,411 & & \multicolumn{3}{|l|}{0,386} & & \\
\hline Tipo almaciguera (TA) & \multicolumn{3}{|c|}{0,231} & 0,755 & & \multicolumn{3}{|l|}{0,513} & & \\
\hline Interacción D x TA & \multicolumn{3}{|c|}{0,343} & 0,327 & & \multicolumn{3}{|l|}{0,731} & & \\
\hline
\end{tabular}


A los 23 ddt la densidad de almaciguera mostró un efecto significativo $(\mathrm{p}<0,05$ ), pero fue independiente del sistema de almácigo. Las plantas provenientes de baja densidad (1144 plantas $\cdot \mathrm{m}^{-2}$ ) presentaron un mayor diámetro del cuello que las de mayor densidad. A los $53 \mathrm{ddt}$, el efecto de densidad de la almaciguera sobre el diámetro del cuello disminuyó, siendo el diámetro de cuello 24,03 mm y 21,3 a 21,7 mm, en las plantas provenientes de almacigueras con baja y alta densidad, respectivamente, a los 84 ddt. Los diámetros de cuello de las plantas, obtenidos a los 23 y a los $53 \mathrm{ddt}$, se indican en el Cuadro 3.

El tamaño de los contenedores, la densidad de plantas y el sistema de almaciguera no afectaron el peso seco radical a los 23 y $53 \mathrm{ddt}$ (Cuadro 3). Esta misma tendencia se mantuvo en las evaluaciones posteriores (datos no presentados).

A los 23 ddt, la densidad de siembra en almaciguera influyó significativamente $(\mathrm{p}<0,05)$ en el peso seco de la canopia (Cuadro 3). Los mejores resultados se obtuvieron con 1144 plantas $\mathrm{m}^{-2}$. Sin embargo, no hubo diferencias entre tipos de almaciguera. A los $53 \mathrm{ddt}$, las plantas provenientes de la menor densidad tuvieron un peso seco de canopia significativamente $(\mathrm{p}<0,05)$ más alto que las plantas producidas en almacigueras con densidades media y alta (Cuadro 3).
La altura de las plantas se determinó midiendo desde la base del cuello hasta el extremo foliar. Este parámetro no se afectó con la densidad ni dependió del sistema de almaciguera usado. A los 53 ddt, la altura de planta varió entre 41,2 y $41,9 \mathrm{~cm}$ en condiciones de baja densidad, de 38,5 a $39,1 \mathrm{~cm}$ en densidad media, y desde 36,4 a 39,2 en alta densidad. La altura promedio de las plantas fue de $58,9 \mathrm{~cm}$ a los $84 \mathrm{ddt}$.

De acuerdo con los resultados obtenidos en el trasplante, la densidad de siembra del almácigo tuvo un efecto significativo $(\mathrm{p}<0,05)$ sobre el diámetro del cuello y peso seco aéreo en plantas evaluadas 53 ddt (Cuadro 3). Esta condición se mantuvo hasta los 114 ddt. No obstante, no hubo diferencias significativas entre los sistemas de almaciguera estudiados.

El diámetro de bulbo no mostró diferencias estadísticas significativas entre densidades de siembra y sistemas de almaciguera. La interacción entre ambos factores no fue significativa (Cuadro 4). A la cosecha el porcentaje cebollas malformadas no presentó diferencias significativas entre los tratamientos, densidad de planta y sistema de almaciguera. Aunque los rendimientos promedios de bulbos exportables decrecieron de menor a mayor densidad, las diferencias obtenidas no fueron estadísticamente significativas. Los efectos de la densidad de planta y tipo de almaciguera en la clasificación de categorías para la obtención
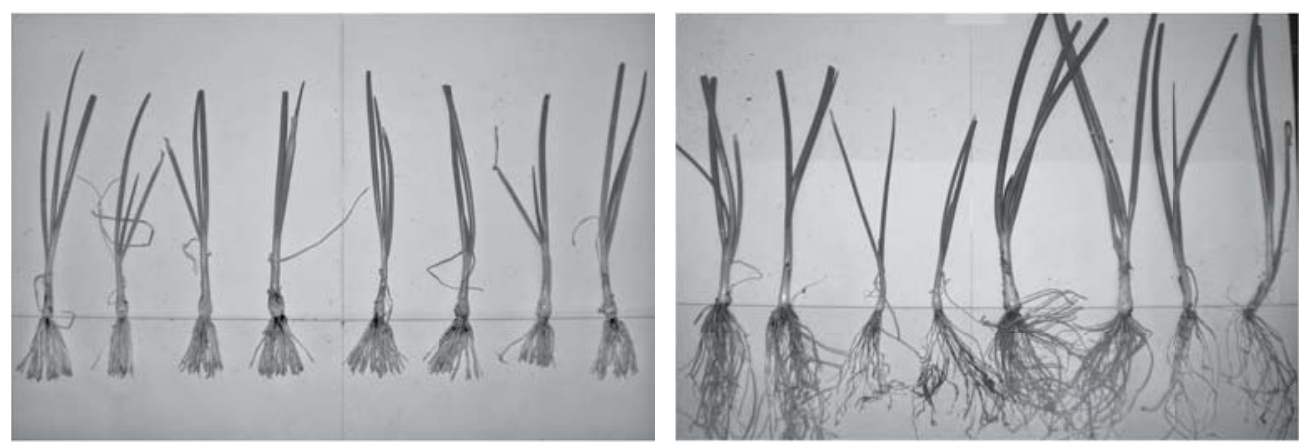

Figura 1. Efecto del tipo de almaciguera, contenedor (izquierda) o cajonera (derecha), sobre plantines de cebolla cv. Grano de Oro a los 77 días después de emergencia. Talagante, 2005.

Figure 1. The effect of the seedling production system, container (left) or boxes (right) on onion plants cv. Grano de Oro, 77 days after emergency. Talagante, 2005. 
de un producto comercial, se presentó en forma independiente, no habiendo respuesta a una interacción de la densidad y sistema de almaciguera (Figura 2).

\section{Discusión}

Según los resultados obtenidos, desde 25 dde el sistema de almaciguera influyó sobre el crecimiento de los plantines. El contenedor favoreció el crecimiento hasta los 40 dde, en cambio la cajonera propició un mayor diámetro del cuello de las cebollas pasado ese período. En consecuencia, el diámetro del cuello de los plantines de cebolla, dependió del sistema de almaciguera y en menor grado por la densidad de siembra. La mejor respuesta se obtuvo en plántulas obtenidas en cajoneras con una densidad de siembra de 1144 planta $\mathrm{m}^{-2}$.

El crecimiento radical de los plantines se afectó a medida que aumentó el tiempo de permanencia en bandejas y cajoneras. Esto fue especialmente evidente en plantas producidas con la mayor densidad, lo que coincide con reportes previos (Wien, 1997). Aunque en el transcurso de todas las mediciones realizadas en la etapa de almaciguera, el peso seco radical no reflejó diferencias entre los dos sistemas de almaciguera, si fue posible apreciar diferencias

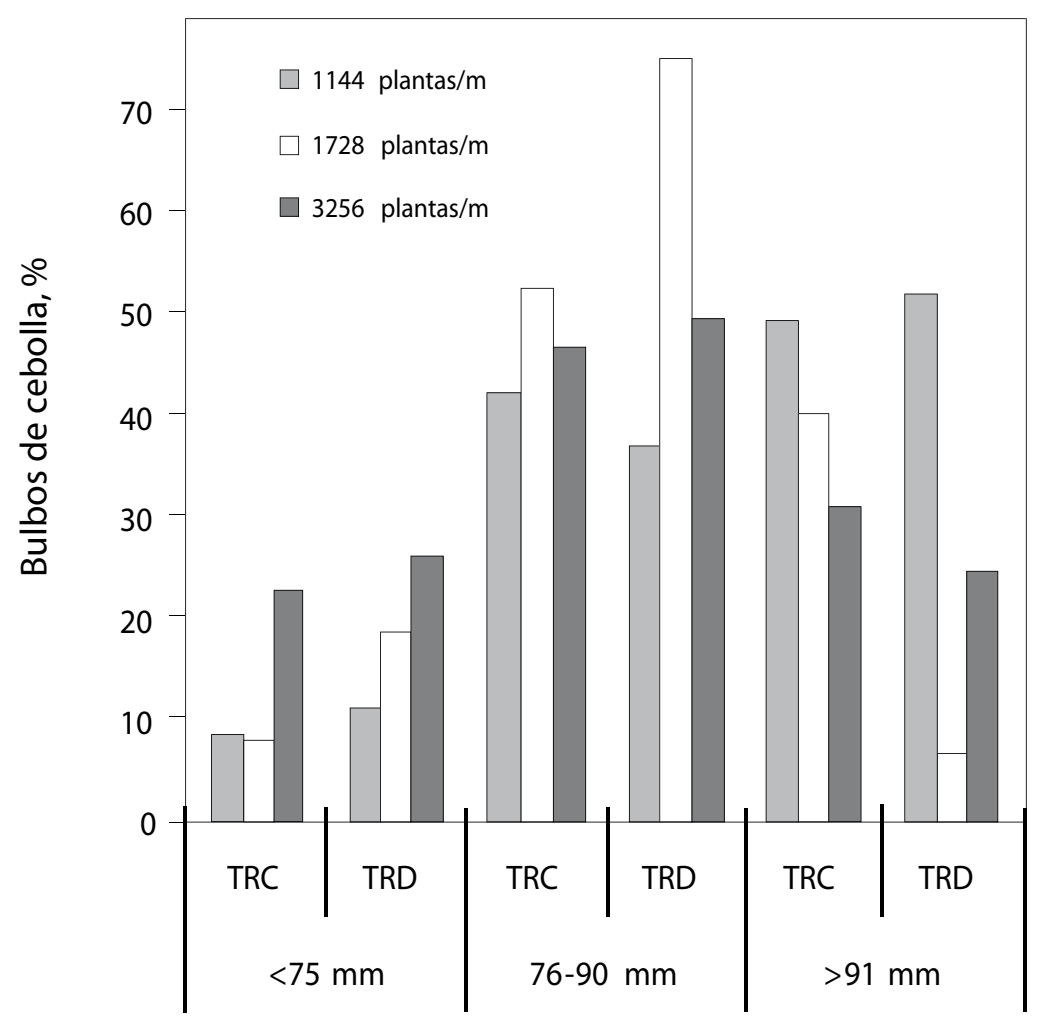

Diámetro del bulbo

Figura 2. Efecto de la densidad y tipo de almaciguera en la distribución (\%) de los calibres de cebollas cv. Grano de Oro según el diámetro del bulbo al momento de la cosecha, 114 días después del transplante. Santiago, 2005. TRC = transplante a raíz cubierta. TRD = transplante a raíz desnuda.

Figure 2. Density and transplant production system effect on the distribution (\%) of onion cv. Grano de Oro sizes, according to bulb diameter at harvest moment, 114 days after transplanting. Santiago, 2005. TRC $=$ covered root transplanting. $T R D=$ bare root transplanting. 
morfológicas. Se detectó visualmente un mayor diámetro y menor largo de raíces en las plantas provenientes de bandejas en comparación con las raíces de las plantas producidas en cajoneras (Figura 1). NeSmith y Duval (1998) indican que los plantines que crecen en alvéolos presentan generalmente una morfología diferente en comparación a las que se desarrollan en condiciones normales de suelo. Según Clark et al. (2003) un impedimento mecánico al crecimiento de raíces, y una consecuente producción de etileno por ellas, podría favorecer un aumento en el diámetro y una menor tasa de elongación. Previamente se ha demostrado que la restricción en el crecimiento de las raíces reduce el peso seco total (follaje y raíces) (Mugnai et al.,2000; Wien, 1997). Similares resultados se obtuvo en este trabajo en la etapa de almaciguera.

Independientemente del sistema de almaciguera empleado, el diámetro del cuello del bulbo durante el cultivo de la cebolla, estuvo influenciado por la densidad de la almaciguera, siendo mayor en plantas provenientes de almácigos con 1144 plantas $/ \mathrm{m}^{2}$. Estos resultados concordaron con los previamente obtenidos por Veiga de Vicenzo y Tessarioli (2003). En forma similar, Vavrina (2002) comprobó que incluso 45 ddt las plantas de tomate en invernadero provenientes de contenedores con alvéolos grandes, presentaron un crecimiento notablemente mayor que plantas obtenidas en alvéolos pequeños.

Finalmente la densidad de siembra y tipo de almaciguera no tuvieron efecto en el rendimiento de bulbos exportables de cebollas. Estos resultados coincidieron con los obtenidos por Reynaud et al. (1999), quienes concluyeron que el rendimiento total de cebolla no depende del volumen de los alvéolos de trasplante. Sin embargo, según estos autores las plántulas provenientes de alvéolos de $4 \mathrm{~cm}^{3}$ produjeron un $16 \%$ menos de bulbos con diámetros mayores a $91 \mathrm{~mm}$, comparado con aquellas obtenidas en alvéolos de $7,1 \mathrm{~cm}^{3}$. Del mismo modo, el tipo de almaciguera y densidad de siembra utilizados en almácigo, no influyó en el porcentaje de malformaciones ni en el rendimiento comercial.
En conclusión, en su primera etapa de crecimiento, las plantas desarrolladas en almácigos de contenedores presentaron una mayor probabilidad de estrés debido a espaciamiento, comparadas con plantas desarrolladas en cajoneras. Hasta los 55 dde, a mayor volumen del contenedor o espaciamiento se favoreció el crecimiento de los plantines. A partir de los 77 dde, se advirtió un efecto negativo en plantas provenientes de bandejas. Una vez transplantados los plantines, la densidad ni el tipo de almaciguera afectó la sobrevivencia en terreno de plantas provenientes de almácigos realizados en contenedores y cajoneras. Las diferencias en el crecimiento de plantas de cebolla provenientes de los distintos sistemas de almacigueras y densidades de siembra perduraron significativamente en el cultivo hasta 53 ddt. Posteriormente, dichas diferencias disminuyeron hasta desaparecer al momento de cosecha. Por lo tanto, es factible producir plantines de cebolla, para transplantar a raíz desnuda o cubierta, en alta densidad o en alvéolos de $5 \mathrm{~cm}^{3}$, sin afectar la calidad final de bulbos cosechados.

\section{Resumen}

Los almácigos de cebolla (Allium cepa) se siembran en suelo o en contenedores (alvéolos) de reducido volumen, en alta densidad, sin considerar el efecto del tamaño del contenedor en el crecimiento del cultivo. Con el propósito de estudiar el efecto del sistema y densidad de la almaciguera se realizaron dos ensayos (almácigo y transplante) en la Región Metropolitana (entre $33,3^{\circ}$ y $34^{\circ}$ lat. sur), Chile. Se empleó cebolla cv. Grano de Oro, cultivar de día largo destinado para guarda, en la temporada 2004-2005. La siembra de almácigos para transplante a raíz cubierta se estudió en contenedores con $286\left(10 \mathrm{~cm}^{3}\right), 432$ $\left(10 \mathrm{~cm}^{3}\right)$ y $814\left(5 \mathrm{~cm}^{3}\right)$ alvéolos, correspondiendo a 1144 (D1), 1728 (D2) y 3256 (D3) plantas $/ \mathrm{m}^{2}$ y para trasplante a raíz desnuda en cajoneras, con iguales densidades, bajo invernadero. Hasta 25 días después de emergencia (dde) la densidad y el tipo de almaciguera no influyeron el diámetro del cuello. A 77 días, D3 presentó un diámetro de cuello de $3,8 \mathrm{~mm} \cdot$ planta $^{-1}$ y D1 $5,2 \mathrm{~mm} \cdot$ planta $^{-1}$; el peso seco (PS) radical fue de 4,8 y 6,2 g.planta ${ }^{-1}$, respectivamente, con 
menor efecto en plantines de contenedores. Los plantines se transplantaron al aire libre, cuando éstos alcanzaron 5 a $6 \mathrm{~mm}$ a nivel de cuello. A los 23 días después del transplante (ddt), hubo diferencias en el diámetro de cuello entre las tres densidades de planta, pero no hubo diferencias significativas entre transplante a raíz cubierta o desnuda. Las plantas provenientes de D3 mostraron menor diámetro de cuello y PS de canopia, hasta 84 ddt. En cosecha no hubo diferencias entre densidades en categorías de calidad y malformaciones de bulbos.

Palabras clave: Almácigo, bandeja, cajonera, densidad de planta, transplante.

\section{Literatura citada}

Aljaro, A. 2001a. Almácigo, producción y selección de plantas y sistemas de plantación. Segundo curso/taller de cebollas. 104 p. Serie Actas $\mathrm{N}^{\circ}$ 11. Instituto de Investigaciones Agropecuarias, Centro Regional de Investigación La Platina (INIA), Santiago, Chile.

Aljaro, A. 2001b. Factores relevantes para la producción de cebollas de exportación. Revista Tierra Adentro (Chile) 40:18-20.
Clark, L.J., Whalley, W.R. and P.B. Barraclough. 2003. How do roots penetrate strong soil? Plant and Soil 255: 93-104.

Marsh, D., and K. Paul. 1988. Influence of container type and cell size on cabbage transplant and field performance. HortScience 23:310-311.

Mugnai, S., P. Vernieri, and F. Tognoni. 2000. Container volume effects on morphology and physiology of tomato seedlings. Acta Horticulturae 516:499-505.

NeSmith, D.S., and J.R. Duval. 1998. The effect of container size. HortTechnology 8:495-498.

Reynaud, M., C. Chauve, J. Gastellu, J. Gounel, D. Leskovar, and C. Vavrina. 1999. Onion growth and yield are influenced by transplant tray cell size and age. Scientia Horticulturae 80:133-143.

Vavrina,C.S. 2002. An introduction to the production of containerized, vegetable transplants. Horticultural Sciences Department, University of Florida, Institute of Food and Agricultural Sciences (IFAS). Fact Sheet HS849.

Veiga de Vincenzo, M.C., and J.Tessarioli. 2003. Onion seedling production in styrofoam trays under controlled environment, as summerplanted onions. Scientia Agricola 60:65-69.

Wien, H.C. 1997.Transplanting. In: Wien, H.C. The Physiology of Vegetable Crops. Reprinted 2002. Cabi Publishing. 662 p. 